\title{
SCATTERING THE GEOMETRY OF WEIGHTED GRAPHS
}

\author{
BATU GÜNEYSU AND MATTHIAS KELLER
}

\begin{abstract}
Given two weighted graphs $\left(X, b_{k}, m_{k}\right), k=1,2$ with $b_{1} \sim b_{2}$ and $m_{1} \sim m_{2}$, we prove a weighted $L^{1}$-criterion for the existence and completeness of the wave operators $W_{ \pm}\left(H_{2}, H_{1}, I_{1,2}\right)$, where $H_{k}$ denotes the natural Laplacian in $\ell^{2}\left(X, m_{k}\right)$ w.r.t. $\left(X, b_{k}, m_{k}\right)$ and $I_{1,2}$ the trivial identification of $\ell^{2}\left(X, m_{1}\right)$ with $\ell^{2}\left(X, m_{2}\right)$. In particular, this entails a very general criterion for the absolutely continuous spectra of $H_{1}$ and $H_{2}$ to be equal.
\end{abstract}

\section{INTRODUCTION}

There is a long history on studying the stability of the absolutely continuous spectrum under various types of perturbations of a sufficiently 'well-behaved' self-adjoint operator $H_{0}$ on a Hilbert space of the form $L^{2}(X, \mu)$. Classical results include perturbations of $H_{0}=-\Delta$ in $L^{2}\left(\mathbb{R}^{m}\right)$ by potentials $V: \mathbb{R}^{m} \rightarrow \mathbb{R}\left[20\right.$, leading to the comparison of $H_{0}$ with $H=-\Delta+V$. Very sophisticated results for perturbations by potentials have been obtained in the one dimensional case $m=1$ and also in the discrete case for Jacobi matrices [4, 14, 15, 21] (see e.g. also [17] and the references therein for perturbations by random potentials).

Another well-studied class of perturbations is provided by replacing the space $X$ by some open subset $U \subset X$ : here one puts some "boundary conditions" on $H_{0}$ in order to obtain the perturbed self-adjoint operator $H=H_{U}$ in $L^{2}(U)$ and compares the absolutely continuous spectra of $H_{0}$ and $H=H_{U}$. Concerning this perturbation theory ('scattering by obstacles'), we mention 22], where so the called Grothendieck factorization principle was introduced as a tool in this context (see also [6, 5]).

A problem in perturbation which is morally rather different from the above problems has been initiated very recently: here, the idea is to assume that the free operator $H_{0}$ and the measure $\mu$ are defined in terms of the 'geometry' of the space $X$. Then, keeping the set $X$ fixed, the inital geometry on $X$ is perturbed, leading to a perturbed operator $H$ that typically lives on a Hilbert space of the form $L^{2}\left(X, \mu^{\prime}\right)$. One then aims to study the absolutely continuous spectrum of $H$ by comparing $H$ with $H_{0}$. Technical problems arise here through the fact that in general one has $\mu \neq \mu^{\prime}$, so that one has to compare operators living in different Hilbert spaces. A prototype of the above situation is provided by Riemannian manifolds: here $X$ is a smooth noncompact manifold which carries a "nice" Riemannian metric $g_{0}$, encoding the free geometry, and the Riemannian metric of interest $g$, encoding the perturbed geometry. One aims to study the absolutely continuous spectrum of the Laplace-Beltrami operator $H=-\Delta_{g}$ acting in $L^{2}\left(X, \mu_{g}\right)$ by that of $H_{0}=-\Delta_{g_{0}}$ acting in $L^{2}\left(X, \mu_{g_{0}}\right)$. A natural approach in this context is provided by studying the associated 
wave operators, which is a genuine two-Hilbert-space scattering problem [18, 8, 1, 1, 9] in this case, as the underlying Riemannian volume measures $\mu_{g_{0}}$ and $\mu_{g}$ are different.

As a contribution to the latter class of problems, in this paper, we aim to study perturbations of the geometry of weighted discrete graphs in terms of the edge and vertex weights. Apart from the work [3] on trees and perturbations of Jacobi matrices (cf. [2] and the references therein), we are not aware of any results in this direction, so that our results should provide the first systematic study of this topic. Moreover, the stability results we obtain include the existence of the wave operators.

Let us be more specific: assume we are given two weighted graphs $\left(X, m_{1}, b_{1}\right)$ and $\left(X, m_{2}, b_{2}\right)$ over the countable set $X$. Let $H_{k}$ denote the natural self-adjoint realization of the weighted Laplacian induced by $\left(X, m_{k}, b_{k}\right)$ in $\ell^{2}\left(X, m_{k}\right)$, defined by means of quadratic forms (cf. Section 2.1 for the precise definitions). If we assume $1 m_{1} \sim m_{2}$, then one has $\ell^{2}\left(X, m_{1}\right)=$ $\ell^{2}\left(X, m_{2}\right)$ and we get the trivial bounded and bijective identification operator

$$
I_{1,2}: \ell^{2}\left(X, m_{1}\right) \longrightarrow \ell^{2}\left(X, m_{2}\right), \quad f \longmapsto f
$$

In this paper we are concerned with finding very general assumptions on the distortion of $m_{2}$ from $m_{1}$ and of $b_{2}$ from $b_{1}$ that imply the existence and completeness of the wave operators

$$
W_{ \pm}\left(H_{2}, H_{1}, I_{1,2}\right)=\underset{t \rightarrow \pm \infty}{\mathrm{st}-\lim _{t \rightarrow \infty}} \exp \left(i t H_{2}\right) I_{1,2} \exp \left(-i t H_{1}\right) \pi_{\mathrm{ac}}\left(H_{1}\right)
$$

In particular, we want to allow arbitrary, possibly locally infinite weighted graphs. As the existence and completeness of the $W_{ \pm}\left(H_{2}, H_{1}, I_{1,2}\right)$ implies that the absolutely continuous spectra of the $H_{k}$ 's are equal, such a result provides a perturbative of calculating new absolutely continuous spectra from known ones, as long as the new weighted geometry does not differ too much from the old one, in a way to be made precise below.

In fact, with the function

$$
m_{1,2}: X \longrightarrow(0, \infty), \quad m_{1,2}(x)=m_{1}(x) / m_{2}(x)
$$

which measures the multiplicative distortion of one vertex weight function from the other, and likewise

$$
b_{1,2}: X \times X \longrightarrow[0, \infty), \quad b_{1,2}(x, y):=\left\{\begin{array}{l}
b_{1}(x, y) / b_{2}(x, y), \text { if } b_{2}(x, y) \neq 0 \\
1, \text { else }
\end{array}\right.
$$

a function that measures the multiplicative distortion of one edge weight function from the other, our main result reads as follows (cf. Theorem 2.1 below):

\footnotetext{
${ }^{1}$ Given a set $A$ and functions $\phi_{1}, \phi_{2}: A \rightarrow[0, \infty)$ we write $\phi_{1} \sim \phi_{2}$, if there exist constants $c, C>0$ such that for all $a \in A$ one has $c \phi_{1}(a) \leq \phi_{2}(a) \leq C \phi_{1}(a)$.
} 
Assume $m_{1} \sim m_{2}, b_{1} \sim b_{2}$, and that there exists $s>0$ such that for both $k=1,2$ one has

$$
\begin{aligned}
& \sum_{x \in X}\left|m_{1,2}(x)^{\frac{1}{2}}-m_{1,2}(x)^{-\frac{1}{2}}\right| \exp \left(-s H_{k}\right)(x, x) m_{k}(x)<\infty, \\
& \sum_{x \in X} \sum_{y \in X}\left|b_{1,2}(x, y)^{\frac{1}{2}}-b_{1,2}(x, y)^{-\frac{1}{2}}\right|\left(\exp \left(-s H_{k}\right)(x, x)+\exp \left(-s H_{k}\right)(y, y)\right) b_{k}(x, y)<\infty .
\end{aligned}
$$

Then the wave operators

$$
W_{ \pm}\left(H_{2}, H_{1}, I\right)=\underset{t \rightarrow \pm \infty}{\mathrm{st}-\lim _{t \rightarrow \infty}} \exp \left(i t H_{2}\right) I_{1,2} \exp \left(-i t H_{1}\right) \pi_{\mathrm{ac}}\left(H_{1}\right)
$$

exist and are complete. Moreover, the $W_{ \pm}\left(H_{2}, H_{1}, I_{1,2}\right)$ are partial isometries with initial space Ran $\pi_{\mathrm{ac}}\left(H_{1}\right)$ and final space Ran $\pi_{\mathrm{ac}}\left(H_{2}\right)$, and one has $\operatorname{spec}_{\mathrm{ac}}\left(H_{1}\right)=\operatorname{spec}_{\mathrm{ac}}\left(H_{2}\right)$.

Using the trivial bounds

$$
\exp \left(-s H_{k}\right)(z, z) \leq 1 / m_{k}(z) \quad \text { for all } s \geq 0, z \in X,
$$

it follows that the assumptions (1.1) are satisfied, if for both $k$ one has

$$
\begin{aligned}
& \sum_{x \in X}\left|m_{1,2}(x)^{\frac{1}{2}}-m_{1,2}(x)^{-\frac{1}{2}}\right|<\infty, \\
& \sum_{x \in X} \sum_{y \in X}\left|b_{1,2}(x, y)^{\frac{1}{2}}-b_{1,2}(x, y)^{-\frac{1}{2}}\right|\left(m_{k}(x)^{-1}+m_{k}(y)^{-1}\right) b_{k}(x, y)<\infty,
\end{aligned}
$$

a criterion which only involves geometric quantities (cf. Corollary 2.2 below).

\section{MAIN RESULTS}

In the sequel, we consider all our spaces of functions to be complex-valued. Let $X$ be a countable set, equipped with its discrete topology. Let $b: X \times X \rightarrow[0, \infty)$ be graph over $X$, that is, $b$ is a symmetric function which is zero on the diagonal and which satisfies

$$
\sum_{y \in X} b(x, y)<\infty \quad \text { for all } y \in X
$$

In other words, all $(x, y)$ with $b(x, y)>0$ are considered as being connected by an edge, weighted according to the value of $b(x, y)$. Considering $b$ as a measure on $X \times X$ in the obvious way (the edge measure), we then get the Hilbert space $\ell^{2}(X \times X, b)$, noting that the scalar pruduct is given by

$$
\langle\alpha, \beta\rangle_{b}=\frac{1}{2} \sum_{X \times X} \alpha \cdot \bar{\beta} \cdot b=\frac{1}{2} \sum_{x, y \in X} \alpha(x, y) \overline{\beta(x, y)} b(x, y) .
$$

We define a nonnegative sesqui-linear form $\mathcal{Q}_{b}$ by means of

$$
\mathcal{Q}_{b}: C_{c}(X) \times C_{c}(X) \longrightarrow \mathbb{C}, \quad \mathcal{Q}_{b}(f, g)=\langle d f, d g\rangle_{b},
$$


with the discrete differential

$$
d: C(X) \longrightarrow C(X \times X), \quad d f(x, y)=f(x)-f(y) .
$$

Given in addition function $m: X \rightarrow(0, \infty)$, again to be considered as a measure on $X$ (the vertex measure), the triple $(X, b, m)$ is called a weighted graph. There is the complex Hilbert space $\ell^{2}(X, m)$ of complex-valued functions on $X$ which are square summable with respect to $m$. Explicitly, the scalar product on $\ell^{2}(X, m)$ is given by

$$
\langle f, g\rangle_{m}=\sum_{X} f \cdot \bar{g} \cdot m=\sum_{x \in X} f(x) \overline{g(x)} m(x) .
$$

Then $\mathcal{Q}_{b}$ is closable in $\ell^{2}(X, m)$ and its closure, a densely defined closed nonnegative sesqui-linear form in $\ell^{2}(X, m)$, is denoted with $Q_{b, m}$. For all $f, g \in \operatorname{Dom}\left(Q_{b, m}\right)$ one has

$$
Q_{b, m}(f, g)=\langle d f, d g\rangle_{b} .
$$

We denote with $H_{b, m}$ the nonnegative self-adjoint operator in $\ell^{2}(X, m)$ corresponding to $Q_{b, m}$. Then one has $\operatorname{Dom}\left(Q_{b, m}\right)=\operatorname{Dom}\left(\sqrt{H_{b, m}}\right)$, and for all $f, g$ in the latter domain of definition one has

$$
Q_{b, m}(f, g)=\left\langle\sqrt{H_{b, m}} f, \sqrt{H_{b, m}} g\right\rangle_{m} .
$$

Consider now two weighted graphs $\left(X, b_{k}, m_{k}\right), k=1,2$, over $X$. We denote the corresponding scalar products, forms and operators by $\langle\cdot, \cdot\rangle_{b_{k}},\langle\cdot, \cdot\rangle_{m_{k}}, Q_{k}:=Q_{b_{k}, m_{k}}$ and $H_{k}:=H_{b_{k}, m_{k}}$. If we assume $m_{1} \sim m_{2}$, then one has $\ell^{2}\left(X, m_{1}\right)=\ell^{2}\left(X, m_{2}\right)$ and there is the trivial bounded and bijective identification operator

$$
I_{1,2}: \ell^{2}\left(X, m_{1}\right) \longrightarrow \ell^{2}\left(X, m_{2}\right), \quad f \longmapsto f .
$$

One has $I_{1,2}^{-1}=I_{2,1}$, and $I_{1,2}^{*} f(x)=m_{1,2}(x) f(x)$, where

$$
m_{1,2}: X \longrightarrow(0, \infty), \quad m_{1,2}(x)=m_{1}(x) / m_{2}(x) .
$$

Define

$$
b_{1,2}: X \times X \longrightarrow[0, \infty), \quad b_{1,2}(x, y):=\left\{\begin{array}{l}
b_{1}(x, y) / b_{2}(x, y), \text { if } b_{2}(x, y) \neq 0 \\
1, \text { else }
\end{array}\right.
$$

Note that $1 / m_{1,2}=m_{2,1}$ and $1 / b_{1,2}=b_{2,1}$, and that if $m_{1} \sim m_{2}$ and $b_{1} \sim b_{2}$, then we get

$$
I_{1,2}\left(\operatorname{Dom}\left(Q_{1}\right)\right)=\operatorname{Dom}\left(Q_{2}\right), \quad\langle\cdot, \cdot\rangle_{b_{2}}=\left\langle b_{1,2}(\cdot), \cdot\right\rangle_{b_{1}} \text {. }
$$

Here comes our main result:

Theorem 2.1. Assume $m_{1} \sim m_{2}, b_{1} \sim b_{2}$, and that there exists $s>0$ such that for both $k=1,2$ one has

$$
\begin{aligned}
& \sum_{x \in X}\left|m_{1,2}(x)^{\frac{1}{2}}-m_{1,2}(x)^{-\frac{1}{2}}\right| \exp \left(-s H_{k}\right)(x, x) m_{k}(x)<\infty \\
& \sum_{x \in X} \sum_{y \in X}\left|b_{1,2}(x, y)^{\frac{1}{2}}-b_{1,2}(x, y)^{-\frac{1}{2}}\right|\left(\exp \left(-s H_{k}\right)(x, x)+\exp \left(-s H_{k}\right)(y, y)\right) b_{k}(x, y)<\infty .
\end{aligned}
$$


Then the wave operators

$$
W_{ \pm}\left(H_{2}, H_{1}, I_{1,2}\right)=\underset{t \rightarrow \pm \infty}{\mathrm{st}-\lim _{t \rightarrow \infty}} \exp \left(i t H_{2}\right) I_{1,2} \exp \left(-i t H_{1}\right) \pi_{\mathrm{ac}}\left(H_{1}\right)
$$

exist and are complete, where $\pi_{\mathrm{ac}}\left(H_{k}\right)$ denotes the projection onto the $H_{k}$-absolutely continuous subspace of $\ell^{2}\left(X, m_{k}\right)$. Moreover, the $W_{ \pm}\left(H_{2}, H_{1}, I_{1,2}\right)$ are partial isometries with inital space $\operatorname{Ran} \pi_{\mathrm{ac}}\left(H_{1}\right)$ and final space $\operatorname{Ran} \pi_{\mathrm{ac}}\left(H_{2}\right)$, and one has $\operatorname{spec}_{\mathrm{ac}}\left(H_{1}\right)=\operatorname{spec}_{\mathrm{ac}}\left(H_{2}\right)$.

Noting that

$$
\exp \left(-s H_{k}\right)(z, z) \leq 1 / m_{k}(z) \quad \text { for all } s \geq 0, z \in X
$$

a trivial consequence of $\exp \left(-s H_{k}\right)$ being a self-adjoint contraction in $\ell^{2}\left(X, m_{k}\right)$, we get:

Corollary 2.2. Assume $m_{1} \sim m_{2}, b_{1} \sim b_{2}$, and that for both $k=1,2$ one has

$$
\begin{aligned}
& \sum_{x \in X}\left|m_{1,2}(x)^{\frac{1}{2}}-m_{1,2}(x)^{-\frac{1}{2}}\right|<\infty \\
& \sum_{x \in X} \sum_{y \in X}\left|b_{1,2}(x, y)^{\frac{1}{2}}-b_{1,2}(x, y)^{-\frac{1}{2}}\right|\left(m_{k}(x)^{-1}+m_{k}(y)^{-1}\right) b_{k}(x, y)<\infty .
\end{aligned}
$$

Then the wave operators

$$
W_{ \pm}\left(H_{2}, H_{1}, I_{1,2}\right)=\underset{t \rightarrow \pm \infty}{\mathrm{st}-\lim } \exp \left(i t H_{2}\right) I_{1,2} \exp \left(-i t H_{1}\right) \pi_{\mathrm{ac}}\left(H_{1}\right)
$$

exist and are complete. Moreover, the $W_{ \pm}\left(H_{2}, H_{1}, I_{1,2}\right)$ are partial isometries with inital space Ran $\pi_{\mathrm{ac}}\left(H_{1}\right)$ and final space Ran $\pi_{\mathrm{ac}}\left(H_{2}\right)$, and one has $\operatorname{spec}_{\mathrm{ac}}\left(H_{1}\right)=\operatorname{spec}_{\mathrm{ac}}\left(H_{2}\right)$.

\section{Applications of Corollary 2.2}

Recall that a weighted graph $(X, b, m)$ is said to have standard weights, if $b$ takes values in $\{0,1\}$ and $m$ is constantly 1 . Observe, that graphs with standard weights are always locally finite. Furthermore, let the uniform closure of the functions with finite support on a discrete set $Y$ be denoted by $C_{0}(Y)$.

Corollary 3.1. Let $\left(X, b_{1}, m_{1}\right)$ be a graph with standard weights and $\beta \in C_{0}(X \times X)$ symmetric, zero on the diagonal and $\mu \in C_{0}(X)$ be such that with

$$
b_{2}:=b_{1}+\beta \quad \text { and } \quad m_{2}:=m_{1}+\mu \text {, }
$$

$b_{2}$ is nonnegative and $m_{2}$ is strictly positive. Let, furthermore, $S_{n} \subseteq X, n \in \mathbb{N}_{0}$, be finite pairwise disjoint subsets such that $X=\bigcup_{n} S_{n}$ and define the averages over $S_{n}$ to be

$$
\beta_{n}:=\frac{1}{\# S_{n}} \sum_{x \in S_{n}, y \in X}|\beta(x, y)| \quad \text { and } \mu_{n}:=\frac{1}{\# S_{n}} \sum_{x \in S_{n}}|\mu(x)|, \quad n \in \mathbb{N}_{0} .
$$

Assume

$$
\sum_{n \in \mathbb{N}_{0}} \# S_{n} \beta_{n}<\infty \quad \text { and } \sum_{n \in \mathbb{N}_{0}} \# S_{n} \mu_{n}<\infty
$$

Then the wave operators

$$
W_{ \pm}\left(H_{2}, H_{1}, I_{1,2}\right)=\underset{t \rightarrow \pm \infty}{\operatorname{st}-\lim } \exp \left(i t H_{2}\right) I_{1,2} \exp \left(-i t H_{1}\right) \pi_{\mathrm{ac}}\left(H_{1}\right)
$$


exist and are complete. Moreover, the $W_{ \pm}\left(H_{2}, H_{1}, I_{1,2}\right)$ are partial isometries with initial space Ran $\pi_{\mathrm{ac}}\left(H_{1}\right)$ and final space Ran $\pi_{\mathrm{ac}}\left(H_{2}\right)$, and one has $\operatorname{spec}_{\mathrm{ac}}\left(H_{1}\right)=\operatorname{spec}_{\mathrm{ac}}\left(H_{2}\right)$.

Proof. Since $\beta$ and $\mu$ are $C_{0}$-functions, there are only finitely many values larger than $1 / 2$ in absolute value. So assume w.l.o.g. $|\beta|,|\mu| \leq 1 / 2$. We calculate using the Taylor expansion of the square root

$$
\begin{aligned}
b_{1,2}^{\frac{1}{2}}-b_{1,2}^{-\frac{1}{2}} & =\frac{(1+\beta)^{1 / 2}-1}{(1+\beta)^{1 / 2}}=\frac{1-\beta^{2}-(1+\beta)^{1 / 2}}{1-\beta^{2}}=-\frac{\beta}{2\left(1-\beta^{2}\right)}+O\left(\beta^{2}\right) \\
m_{1,2}^{\frac{1}{2}}-m_{1,2}^{-\frac{1}{2}} & =-\frac{\mu}{2\left(1-\mu^{2}\right)}+O\left(\mu^{2}\right),
\end{aligned}
$$

where we use the Landau symbol $O$ for real valued functions on a discrete sets $Y$ with respect to the limit to the point $\infty_{Y}$ in the Alexandrov compactification $Y \cup\left\{\infty_{Y}\right\}$ of $Y$. Hence,

$$
\sum_{x \in X}\left|m_{1,2}(x)^{\frac{1}{2}}-m_{1,2}(x)^{-\frac{1}{2}}\right| \leq \sum_{n \in \mathbb{N}_{0}} \# S_{n}\left(\mu_{n}+O\left(\mu_{n}^{2}\right)\right)<\infty .
$$

Since we assumed $|\beta|,|\mu| \leq 1 / 2$, we infer $m_{k}^{-1}, b_{k} \leq 2, k=1,2$ and, therefore,

$$
\sum_{x, y \in X}\left|b_{1,2}(x, y)^{\frac{1}{2}}-b_{1,2}(x, y)^{-\frac{1}{2}}\right|\left(m_{k}(x)^{-1}+m_{k}(y)^{-1}\right) b_{k}(x, y) \leq \sum_{n \in \mathbb{N}_{0}} \# S_{n}\left(\beta_{n}+O\left(\beta_{n}^{2}\right)\right)<\infty .
$$

Thus, we deduce the statement by Corollary 2.2 .

Next, we discuss some particular examples where we can apply the corollary above directly.

Example 3.2 (The Euclidean lattice). Consider the Laplacian $H_{1}$ with standard weights on $\mathbb{Z}^{d}$. It is folklore that the spectrum of this operator is purely absolutely continuous

$$
\operatorname{spec}\left(H_{1}\right)=\operatorname{spec}_{\mathrm{ac}}\left(H_{1}\right)=[0,4 d]
$$

which can be proven using the discrete Fourier transform. Denote the distance spheres with respect to the supremum norm on $\mathbb{Z}^{d}$ by $S_{n}, n \in \mathbb{N}_{0}$. Then,

$$
\# S_{n} \sim n^{d-1}
$$

and all $\beta \in C_{0}\left(\mathbb{Z}^{d} \times \mathbb{Z}^{d}\right)$ and $\mu \in C_{0}\left(\mathbb{Z}^{d}\right)$ with

$$
\beta_{n}, \mu_{n} \in O\left(n^{-(d+\varepsilon)}\right)
$$

for some $\varepsilon>0$ satisfy the assumptions of Corollary [3.1, in particular, for those $\beta, \mu$ one has

$$
\operatorname{spec}\left(H_{2}\right)=\operatorname{spec}_{\mathrm{ac}}\left(H_{1}\right)=[0,4 d] .
$$

For Cayley graphs of abelian groups it is also known that the Laplacian has purely continuous spectrum, [10]. These graphs have polynomial growth and therefore every $\beta, \mu$ that decays a bit more than one order of the polynomial growth satisfies the assumptions of Corollary 3.1, showing that the correspoding weighted Laplacians still have a purely continuous spectrum. 
Example 3.3 (Regular trees). Consider the Laplacian $H_{1}$ with standard weights on a $k$-regular tree. The spectrum of this operator is purely absolutely continuous

$$
\operatorname{spec}\left(H_{1}\right)=\operatorname{spec}_{\mathrm{ac}}(H)=[2(k+1)-2 \sqrt{k}, 2(k+1)+2 \sqrt{k}] .
$$

This is also folklore and attributed to Furstenberg. It can be seen using the recursion formula of the Green's function to compute the spectral density, see [16]. Denote the distance spheres with respect to the combinatorial graph distance by $S_{n}, n \in \mathbb{N}$. Then, \# $S_{n}=k^{n}$, and we may allow for

$$
\beta_{n}, \mu_{n} \in O\left(n^{-(1+\varepsilon)} k^{-n}\right)
$$

for some $\varepsilon>0$ to apply Corollary 3.1.

Example 3.4 (Trees of finite cone type and Galton Watson trees). A tree of finite cone type, [19], is generated by substitution matrix $M \in \mathbb{N}_{0}^{N \times N}$ for some natural number $N$. Starting with a root for which one has to pick a label from $\{1, \ldots, N\}$ every vertex with label $k$ has $M_{k, l}$ forward neighbors of label $l$. The Laplacian on these trees has finitely many intervals of pure absolutely continuous spectrum provided $M$ is irreducible and has positive diagonal, [12, 13. Denote again the distance spheres with respect to the combinatorial graph distance by $S_{n}, n \in \mathbb{N}_{0}$. Then, by elementary considerations one has $\# S_{n} \sim \gamma^{n}$, where $\gamma$ is the largest eigenvalue of $M$. Hence, we may allow for

$$
\beta_{n}, \mu_{n} \in O\left(n^{-(1+\varepsilon)} \gamma^{-n}\right)
$$

for some $\varepsilon>0$ to apply Corollary 3.1.

These considerations may be generalized to certain Galton-Watson trees. In [11] it is shown that if the distribution of such a tree is close to a deterministic tree of finite cone type, then the Laplacians shares most of the absolutely continuous spectrum of the deterministic tree. By the arguments above we may perturb the weights on these random trees according to the allowed perturbations for the corresponding tree of finite cone type to ensure stability of the absolutely continuous spectrum.

\section{Proof of Theorem 2.1}

Assume we are given two weighted graphs $\left(X, b_{k}, m_{k}\right), k=1,2$, over $X$. With

$$
\tilde{m}_{1,2}:=\sqrt{m_{1,2}}-1 / \sqrt{m_{1,2}}, \quad \tilde{b}_{1,2}:=\sqrt{b_{1,2}}-1 / \sqrt{b_{1,2}},
$$

we define maximally defined multiplication operators

$$
\begin{aligned}
& S_{1,2 ; k}: \ell^{2}\left(X, m_{k}\right) \longrightarrow \ell^{2}\left(X, m_{k}\right), \quad S_{1,2 ; k} f(x)=\left|\tilde{m}_{1,2}(x)\right|^{\frac{1}{2}} f(x), \\
& \widehat{S_{1,2 ; k}}: \ell^{2}\left(X \times X, b_{k}\right) \longrightarrow \ell^{2}\left(X \times X, b_{k}\right), \quad \widehat{S_{1,2 ; k}} \alpha(x, y)=\left|\tilde{b}_{1,2}(x, y)\right|^{\frac{1}{2}} \alpha(x, y), \\
& U_{1,2}: \ell^{2}\left(X, m_{1}\right) \longrightarrow \ell^{2}\left(X, m_{2}\right), \quad U_{1,2} f(x)=\operatorname{sgn}\left(\tilde{m}_{1,2}(x)\right) m_{1,2}(x)^{-\frac{1}{2}} f(x), \\
& \widehat{U_{1,2}}: \ell^{2}\left(X \times X, b_{1}\right) \longrightarrow \ell^{2}\left(X \times X, b_{2}\right), \quad \widehat{U_{1,2}} \alpha(x, y)=\operatorname{sgn}\left(\tilde{b}_{1,2}(x, y)\right) b_{1,2}(x, y)^{-\frac{1}{2}} \alpha(x, y) .
\end{aligned}
$$


The operators $U_{1,2}, \widehat{U_{1,2}}$ are always unitary, and the operators $S_{1,2 ; k}, \widehat{S_{1,2 ; k}}$ are always selfadjoint and in addition bounded if $m_{1} \sim m_{2}$ and $b_{1} \sim b_{2}$. Denote with $d_{k}$ the closure of the operator $d$ considered as an operator from $\ell^{2}\left(X, m_{k}\right)$ to $\ell^{2}\left(X \times X, b_{k}\right)$, defined initially on finitely supported functions. Then one has $H_{k}=d_{k}^{*} d_{k}$, with $\operatorname{Dom}\left(d_{k}\right)=\operatorname{Dom}\left(\sqrt{H_{k}}\right)=$ $\operatorname{Dom}\left(Q_{k}\right)$, by Proposition B.1. We denote the heat semigroups with

$$
P_{s}^{(k)}:=\exp \left(-s H_{k}\right) \in \mathscr{L}\left(\ell^{2}\left(X, m_{k}\right)\right), \quad s>0,
$$

and we further define

$$
\hat{P}_{s}^{(k)}:=d_{k} P_{s}^{(k)} \in \mathscr{L}\left(\ell^{2}\left(X, m_{k}\right), \ell^{2}\left(X \times X, b_{k}\right)\right), \quad s>0 .
$$

In view of $\hat{P}_{s}^{(k)}=d_{k} \exp \left(-s d_{k}^{*} d_{k}\right)$, the asserted boundedness of $\hat{P}_{s}^{(k)}$ follows again from Proposition B.1.

The main idea for the proof of Theorem 2.1 is to use the above constructions in order to write the operator $T$ from the abstract Belopol'skii-Birman Theorem (cf. Section A) essentially as a sum of operators of the form

$$
\left(A_{1} \hat{P}_{s}^{(2)}\right)^{*} X_{1} A_{2} \hat{P}_{s}^{(1)} X_{2}, \quad\left(A_{3} P_{s}^{(2)}\right)^{*} X_{3} A_{4} P_{s}^{(1)} X_{4}
$$

where the $X_{j}$ are bounded and where the multiplication operators $A_{j}$ reflect the deviations $m_{1,2}$ and $b_{1,2}$ and will be chosen in a way that

$$
\left(A_{1} \hat{P}_{s}^{(2)}\right)^{*} X_{1} A_{2} \hat{P}_{s}^{(1)} X_{2}, \quad\left(A_{3} P_{s}^{(2)}\right)^{*} X_{3} A_{4} P_{s}^{(1)} X_{4}
$$

will be Hilbert-Schmidt. This idea stems from the scattering theory of Riemannian manifolds [8, 7, 1] and has been called Hempel-Post-Weder formula in [7]:

Lemma 4.1 (Discrete HPW formula). Assume $m_{1} \sim m_{2}, b_{1} \sim b_{2}, s>0$. Then defining the bounded operator $T_{1,2, s}: \ell^{2}\left(X, m_{1}\right) \rightarrow \ell^{2}\left(X, m_{2}\right)$ by

$$
T_{1,2, s}:=\left(\widehat{S_{1,2 ; 2}} \widehat{P}_{s}^{(2)}\right)^{*} \widehat{U_{1,2}} \widehat{S_{1,2 ; 1}} \widehat{P}_{s}^{(1)}-\left(S_{1,2 ; 2} P_{s}^{(2)}\right)^{*} U_{1,2} S_{1,2 ; 1} P_{s / 2}^{(1)} H_{1} P_{s / 2}^{(1)},
$$

the following formula holds for all $f_{2} \in \operatorname{Dom}\left(H_{2}\right), f_{1} \in \operatorname{Dom}\left(H_{2}\right)$,

$$
\left\langle f_{2}, T_{1,2, s} f_{1}\right\rangle_{m_{2}}=\left\langle H_{2} f_{2}, P_{s}^{(2)} I_{1,2} P_{s}^{(1)} f_{1}\right\rangle_{m_{2}}-\left\langle f_{2}, P_{s}^{(2)} I_{1,2} P_{s}^{(1)} H_{1} f_{1}\right\rangle_{m_{2}} .
$$

Proof. Adding and subtracting the term $\left\langle I_{2,1} f_{2}, H_{1} P_{s}^{(1)} f_{1}\right\rangle_{m_{1}}$ we get

$$
\begin{aligned}
\langle & \left.H_{2} f_{2}, P_{s}^{(2)} I_{1,2} P_{s}^{(1)} f_{1}\right\rangle_{m_{2}}-\left\langle f_{2}, P_{s}^{(2)} I_{1,2} P_{s}^{(1)} H_{1} f_{1}\right\rangle_{m_{2}} \\
= & \left\langle H_{2} P_{s}^{(2)} f_{2}, I_{1,2} P_{s}^{(1)} f_{1}\right\rangle_{m_{2}}-\left\langle I_{2,1} P_{s}^{(2)} f_{2}, H_{1} P_{s}^{(1)} f_{1}\right\rangle_{m_{1}} \\
& -\left\langle P_{s}^{(2)} f_{2},\left(I_{1,2}-I_{2,1}^{*}\right) H_{1} P_{s}^{(1)} f_{1}\right\rangle_{m_{1}} \\
= & \left\langle d_{2} P_{s}^{(2)} f_{2}, d_{2} I_{1,2} P_{s}^{(1)} f_{1}\right\rangle_{b_{2}}-\left\langle d_{1} I_{2,1} P_{s}^{(2)} f_{2}, d_{1} P_{s}^{(1)} f_{1}\right\rangle_{b_{1}} \\
& -\left\langle P_{s}^{(2)} f_{2},\left(I_{1,2}-I_{2,1}^{*}\right) H_{1} P_{s}^{(1)} f_{1}\right\rangle_{m_{2}}
\end{aligned}
$$

Using $\langle\cdot, \cdot\rangle_{b_{1}}=\left\langle b_{1,2}^{-1}(\cdot), \cdot\right\rangle_{b_{1}}$ and

$$
1-b_{1,2}^{-1}=b_{1,2}^{-\frac{1}{2}}\left(b_{1,2}^{\frac{1}{2}}-b_{1,2}^{-\frac{1}{2}}\right)=b_{1,2}^{-\frac{1}{2}} \tilde{b}_{1,2}=b_{1,2}^{-\frac{1}{2}}\left|\tilde{b}_{1,2}\right| \operatorname{sgn}\left(\tilde{b}_{1,2}\right),
$$


the first two terms combine to

$$
\begin{aligned}
& \left\langle d_{2} P_{s}^{(2)} f_{2}, d_{2} I_{1,2} P_{s}^{(1)} f_{1}\right\rangle_{b_{2}}-\left\langle d_{1} I_{2,1} P_{s}^{(2)} f_{2}, d_{1} P_{s}^{(1)} f_{1}\right\rangle_{b_{1}} \\
& =\sum_{X \times X} d P_{s}^{(2)} f_{2} \cdot \overline{d P_{s}^{(1)} f_{1}} \cdot b_{2} \cdot\left(1-b_{1,2}^{-1}\right) \\
& =\sum_{X \times X} d P_{s}^{(2)} f_{2} \cdot \overline{\left|\tilde{b}_{1,2}\right|^{\frac{1}{2}} \cdot \operatorname{sgn}\left(\tilde{b}_{1,2}\right) b_{1,2}^{-\frac{1}{2}} \cdot\left|\tilde{b}_{1,2}\right|^{\frac{1}{2}} \cdot d P_{s}^{(1)} f_{1}} \cdot b_{2} \\
& =\left\langle\widehat{P}_{s}^{(2)} f_{2}, \widehat{S}_{1,2 ; 2} \widehat{U}_{1,2} \widehat{S}_{1,2 ; 1} \widehat{P}_{s}^{(1)} f_{1}\right\rangle_{b_{2}} \\
& =\left\langle f_{2},\left(\widehat{P}_{s}^{(2)}\right)^{*} \widehat{S}_{1,2 ; 2} \widehat{U}_{1,2} \widehat{S}_{1,2 ; 1} \widehat{P}_{s}^{(1)} f_{1}\right\rangle_{b_{2}} .
\end{aligned}
$$

Likewise, one has

$$
\begin{aligned}
& \left\langle P_{s}^{(2)} f_{2},\left(I_{1,2}-I_{2,1}^{*}\right) H_{1} P_{s}^{(1)} f_{1}\right\rangle_{m_{2}} \\
& =\sum_{X} P_{s}^{(2)} f_{2} \cdot\left(1-m_{1,2}^{-1}\right) \cdot \overline{H_{1} P_{s}^{(1)} f_{1}} \cdot m_{2} \\
& =\sum_{X} P_{s}^{(2)} f_{2} \cdot \overline{\left|\tilde{m}_{1,2}\right|^{\frac{1}{2}} \cdot \operatorname{sgn}\left(\tilde{m}_{1,2}\right) m_{1,2}^{-\frac{1}{2}} \cdot\left|\tilde{m}_{1,2}\right|^{\frac{1}{2}} H_{1} P_{s}^{(1)} f_{1}} \cdot m_{2} \\
& =\left\langle f_{2}, P_{s}^{(2)} S_{1,2 ; 2} U_{1,2} S_{1,2 ; 1} H_{2} P_{s}^{(1)} f_{1}\right\rangle_{m_{2}} \\
& =\left\langle f_{2}, P_{s}^{(2)} S_{1,2 ; 2} U_{1,2} S_{1,2 ; 1} P_{s / 2}^{(1)} H_{2} P_{s / 2}^{(1)} f_{1}\right\rangle_{m_{2}},
\end{aligned}
$$

completing the proof.

Lemma 4.2. For every $s>0$ the operator

$$
\widehat{P}_{s}^{(k)}=d_{k} P_{s}^{(k)} \in \mathscr{L}\left(\ell^{2}\left(X, m_{k}\right), \ell^{2}\left(X \times X, b_{k}\right)\right)
$$

is an integral operator having a uniquely determined integral kernel

$$
\hat{P}_{s}^{(k)}(\cdot, \cdot, \cdot): X \times X \times X \longrightarrow \mathbb{C}
$$

which for all $f \in \ell^{2}\left(X, m_{k}\right), x, y \in X$ satisfies

$$
\begin{aligned}
& \hat{P}_{s}^{(k)} f(x, y)=\sum_{z \in X} \hat{P}_{s}^{(k)}(x, y, z) f(z) m_{k}(z) \\
& \sum_{z \in X}\left|\hat{P}_{s}^{(k)}(x, y, z)\right|^{2} m_{k}(z) \leq 2 P_{2 s}^{(k)}(x, x)+2 P_{2 s}^{(k)}(y, y) .
\end{aligned}
$$

Proof. By Riesz-Fischer's duality theorem it suffices to prove that for all $f \in \ell^{2}\left(X, m_{k}\right)$ and all $x, y \in X$ one has

$$
\left|\hat{P}_{s}^{(k)} f(x, y)\right| \leq\left(P_{2 s}^{(k)}(x, x)^{1 / 2}+P_{2 s}^{(k)}(y, y)^{1 / 2}\right)\|f\|_{m_{k}},
$$


showing that for fixed $s, x, y$ the linear functional $f \mapsto \hat{P}_{s}^{(k)} f(x, y)$ on $\ell^{2}\left(X, m_{k}\right)$ is bounded with bound

$$
\leq P_{2 s}^{(k)}(x, x)^{1 / 2}+P_{2 s}^{(k)}(y, y)^{1 / 2}
$$

The latter estimate follows from the trivial bound

$$
\left|\hat{P}_{s}^{(k)} f(x, y)\right|=\left|d P_{s}^{(k)} f(x, y)\right| \leq\left|P_{s}^{(k)} f(x)\right|+\left|P_{s}^{(k)} f(y)\right|
$$

and

$$
\begin{aligned}
& \left|P_{s}^{(k)} f(z)\right| \leq \sum_{z^{\prime} \in X} P_{s}^{(k)}\left(z, z^{\prime}\right)\left|f\left(z^{\prime}\right)\right| m_{k}\left(z^{\prime}\right) \\
& \leq\left(\sum_{z^{\prime} \in X} P_{s}^{(k)}\left(z, z^{\prime}\right)^{2} m_{k}\left(z^{\prime}\right)\right)^{1 / 2}\|f\|_{m_{k}}=\sqrt{P_{2 s}^{(k)}(z, z)}\|f\|_{m_{k}}
\end{aligned}
$$

by the semigroup property.

Using Belopol'skii-Birman's theorem (cf. section $\mathrm{A}$ below) we can now give the

Proof of Theorem [2.1. Firstly, as we have noted $m_{1} \sim m_{2}$ and $b_{1} \sim b_{2}$ implies

$$
I_{1,2}\left(\operatorname{Dom}\left(Q_{1}\right)\right)=\operatorname{Dom}\left(Q_{2}\right) \text {. }
$$

The operator

$$
A:=\left(I_{1,2}^{*} I_{1,2}-\mathrm{id}_{\ell^{2}\left(X, m_{1}\right)}\right) P_{s}^{(1)}
$$

is Hilbert-Schmidt, and thus compact: As the function $r \mapsto P_{r}^{(1)}(x, x)$ is monotonously decreasing, this follows from

$$
A(x, y)=\left(m_{1,2}(x)-1\right) P_{s}^{(1)}(x, y), \quad \sum_{y \in X} P_{s}^{(1)}(x, y)^{2} m_{1}(y) \leq P_{2 s}^{(1)}(x, x),
$$

and thus

$$
\begin{aligned}
& \sum_{x \in X} \sum_{y \in Y}|A(x, y)|^{2} m_{1}(y) m_{1}(x) \leq \sum_{x \in X}\left|m_{1,2}(x)-1\right|^{2} P_{s / 2}^{(1)}(x, x) m_{1}(x) \\
& \leq C \sum_{x \in X}\left|m_{1,2}(x)^{\frac{1}{2}}-m_{1,2}(x)^{-\frac{1}{2}}\right| P_{s / 2}^{(1)}(x, x) m_{1}(x)<\infty
\end{aligned}
$$

noting that by $m_{1} \sim m_{2}$ and $1 / m_{1,2}=m_{2,1}$ the functions $m_{1,2}$ and $1 / m_{1,2}$ are bounded. The proof is finished, once we have shown that the operator

$$
T_{1,2, s}=\left(\widehat{S_{1,2 ; 2}} \widehat{P}_{s}^{(2)}\right)^{*} \widehat{U_{1,2}} \widehat{S_{1,2 ; 1}} \widehat{P}_{s}^{(1)}-\left(S_{1,2 ; 2} P_{s}^{(2)}\right)^{*} U_{1,2} S_{1,2 ; 1} P_{s / 2}^{(1)} H_{1} P_{s / 2}^{(1)},
$$

from Lemma 4.1 is trace class. To see this, note that the product of Hilbert-Schmidt operators is trace class, that the product of a bounded operator and a Hilbert-Schmidt operator is Hilbert-Schmidt, and that adjoints of Hilbert-Schmidt operators also have this property. Thus, since $U_{1,2}, \widehat{U}_{1,2}$ are unitary and $H_{1} P_{s / 2}^{(1)}$ is bounded, it suffices to prove that the operators

$$
B_{k}:=\widehat{S_{1,2 ; k}} \widehat{P}_{s}^{(k)}, \quad k=1,2
$$


and

$$
C_{1}:=S_{1,2 ; 1} P_{s / 2}^{(1)}, \quad C_{2}:=S_{1,2 ; 2} P_{s}^{(2)}
$$

are Hilbert-Schmidt. For the former, this follows from

$$
\begin{aligned}
& B_{k}(x, y, z)=\left|b_{1,2}(x)^{\frac{1}{2}}(x, y)-b_{1,2}(x, y)^{-\frac{1}{2}}\right|^{\frac{1}{2}} \widehat{P}_{s}^{(k)}(x, y, z), \\
& \sum_{z \in X}\left|\widehat{P}_{s}^{(k)}(x, y, z)\right|^{2} m_{k}(z) \leq 2 P_{2 s}^{(k)}(x, x)+2 P_{2 s}^{(k)}(y, y),
\end{aligned}
$$

where we have used Lemma 4.2, and so by the assumptions of the theorem

$$
\begin{aligned}
& \sum_{x \in X} \sum_{y \in X} \sum_{z \in X}\left|B_{k}(x, y, z)\right|^{2} m_{k}(z) b_{k}(x, y) \\
& \leq 2 \sum_{x \in X} \sum_{y \in X} \sum_{z \in X}\left|b_{1,2}(x)^{\frac{1}{2}}(x, y)-b_{1,2}(x, y)^{-\frac{1}{2}}\right|\left(P_{s / 2}^{(k)}(x, x)+P_{s / 2}^{(k)}(y, y)\right) b_{k}(x, y)<\infty .
\end{aligned}
$$

The Hilbert-Schmidt property of $C_{2}$ follows from

$$
C_{2}(x, y)=\mid m_{1,2}(x)^{\frac{1}{2}}-m_{1,2}(x)^{-\left.\frac{1}{2}\right|^{\frac{1}{2}}} P_{s}^{(2)}(x, y), \quad \sum_{y \in X} P_{s}^{(2)}(x, y) m_{2}(y) \leq P_{2 s}^{(2)}(x, x),
$$

and so by assumptions of the theorem

$$
\sum_{x \in X} \sum_{y \in X}\left|C_{2}(x, y)\right|^{2} m_{2}(x) m_{2}(y) \leq \sum_{x \in X}\left|m_{1,2}(x)^{\frac{1}{2}}-m_{1,2}(x)^{-\frac{1}{2}}\right| P_{s / 2}^{(2)}(x, x) m_{2}(x)<\infty .
$$

Likewise one finds

$$
\sum_{x \in X} \sum_{y \in X}\left|C_{1}(x, y)\right|^{2} m_{1}(x) m_{1}(y) \leq \sum_{x \in X}\left|m_{1,2}(x)^{\frac{1}{2}}-m_{1,2}(x)^{-\frac{1}{2}}\right| P_{s / 2}^{(1)}(x, x) m_{1}(x)<\infty,
$$

finishing the proof of Theorem 2.1.

\section{APPENDIX A. Belopol'ski-BIRMAN THEOREM}

Theorem A.1. (Belopol'skii-Birman) For $k=1,2$, let $H_{k} \geq 0$ be a self-adjoint operator in a complex Hilbert space $\mathscr{H}_{k}$, where in the sequel $\pi_{\mathrm{ac}}\left(H_{k}\right)$ denotes the projection onto the $H_{k}$-absolutely continuous subspace of $\mathscr{H}_{k}$. Assume that $I \in \mathscr{L}\left(\mathscr{H}_{1}, \mathscr{H}_{2}\right)$ is such that the following assumptions hold:

- I has a two-sided bounded inverse

- One has either $I\left(\operatorname{Dom}\left(\sqrt{H_{1}}\right)\right)=\operatorname{Dom}\left(\sqrt{H_{2}}\right)$ or $I\left(\operatorname{Dom}\left(H_{1}\right)\right)=\operatorname{Dom}\left(H_{2}\right)$.

- The operator

$$
\left(I^{*} I-\operatorname{id}_{\mathscr{H}_{1}}\right) \exp \left(-r H_{1}\right): \mathscr{H}_{1} \rightarrow \mathscr{H}_{1} \text { is compact for some } r>0 .
$$

- There exists a trace class operator $T: \mathscr{H}_{1} \rightarrow \mathscr{H}_{2}$ and a number $s>0$ such that for all $f_{2} \in \operatorname{Dom}\left(H_{2}\right), f_{1} \in \operatorname{Dom}\left(H_{1}\right)$ one has

$$
\begin{aligned}
\left\langle f_{2}, T f_{1}\right\rangle_{\mathscr{H}_{2}}= & \left\langle H_{2} f_{2}, \exp \left(-s H_{2}\right) I \exp \left(-s H_{1}\right) f_{1}\right\rangle_{\mathscr{H}_{2}} \\
& -\left\langle f_{2}, \exp \left(-s H_{2}\right) I \exp \left(-s H_{1}\right) H_{1} f_{1}\right\rangle_{\mathscr{H}_{2}} .
\end{aligned}
$$


Then the wave operators

$$
W_{ \pm}\left(H_{2}, H_{1}, I\right)=\underset{t \rightarrow \pm \infty}{\mathrm{st}-\lim _{\mathrm{m}}} \exp \left(i t H_{2}\right) I \exp \left(-i t H_{1}\right) \pi_{\mathrm{ac}}\left(H_{1}\right)
$$

exis 2 $^{2}$ and are complete, where completeness means that

$$
\left(\operatorname{Ker} W_{ \pm}\left(H_{2}, H_{1}, I\right)\right)^{\perp}=\operatorname{Ran} \pi_{\mathrm{ac}}\left(H_{1}\right), \quad \overline{\operatorname{Ran} W_{ \pm}\left(H_{2}, H_{1}, I\right)}=\operatorname{Ran} \pi_{\mathrm{ac}}\left(H_{2}\right) .
$$

Moreover, $W_{ \pm}\left(H_{2}, H_{1}, I\right)$ are partial isometries with inital space Ran $\pi_{\mathrm{ac}}\left(H_{1}\right)$ and final space Ran $\pi_{\mathrm{ac}}\left(H_{2}\right)$, and one has $\operatorname{spec}_{\mathrm{ac}}\left(H_{1}\right)=\operatorname{spec}_{\mathrm{ac}}\left(H_{2}\right)$.

Proof. This result is essentially included in Theorem XI.13 from [20]. A detailed proof is given in [7].

\section{Appendix B. Some facts on quadratic forms in Hilbert spaces}

The following result is certainly well-known. As we have not been able to find a precise reference, we have included a quick proof:

Proposition B.1. Let $D$ be a densely defined, closed operator from a Hilbert space $\mathscr{H}$ to another Hilbert space $\widetilde{\mathscr{H}}$. Then the following assertions hold:

a) The nonnegative, densely defined sesquilinear form $Q_{D}$ in $\mathscr{H}$ given by

$$
Q_{D}\left(f_{1}, f_{2}\right):=\left\|D f_{1}\right\|^{2}, \quad \operatorname{Dom}\left(Q_{D}\right)=\operatorname{Dom}(D)
$$

is closed, and its associated nonnegative self-adjoint operator is $D^{*} D$.

b) For all $t>0$ the operator $D \exp \left(-t D^{*} D\right)$ from $\mathscr{H}$ to $\widetilde{\mathscr{H}}$ is in $\mathscr{L}(\mathscr{H}, \widetilde{\mathscr{H}})$.

Proof. a) It is checked easily that $Q_{D}$ is closed. Let $H_{D} \geq 0$ denote its associated selfadjoint operator. If $f_{1} \in \operatorname{Dom}\left(H_{D}\right)$, then we have $f_{1} \in \operatorname{Dom}\left(Q_{D}\right)=\operatorname{Dom}(D)$, and for all $f_{2} \in \operatorname{Dom}(D)$

$$
\left\langle H_{D} f_{1}, f_{2}\right\rangle=Q_{D}\left(f_{1}, f_{2}\right)=\left\langle D f_{1}, D f_{2}\right\rangle
$$

which implies $D f_{1} \in \operatorname{Dom}\left(D^{*}\right)$ and $D^{*} D f_{1}=H_{D} f_{1}$. Conversely, if $f_{1} \in \operatorname{Dom}(D)=$ $\operatorname{Dom}\left(Q_{D}\right)$ with $D f_{1} \in \operatorname{Dom}\left(D^{*}\right)$, then for all $f_{2} \in \operatorname{Dom}(D)=\operatorname{Dom}\left(Q_{D}\right)$ we have

$$
Q_{D}\left(f_{1}, f_{2}\right)=\left\langle D f_{1}, D f_{2}\right\rangle=\left\langle D^{*} D f_{1}, f_{2}\right\rangle
$$

which implies $f_{1} \in \operatorname{Dom}\left(H_{D}\right)$ and $H_{D} f_{1}=D^{*} D f_{1}$.

b) Set $H_{D}:=D^{*} D$. The polar decomposition of $D$ reads $D=U \sqrt{H_{D}}$, where $U$ is an everywhere defined operator from $\mathscr{H}$ to $\widetilde{\mathscr{H}}$ which maps

$$
\overline{\operatorname{Ran}\left(\sqrt{H_{D}}\right)} \longrightarrow \overline{\operatorname{Ran}(D)} \text { isometrically. }
$$

Thus, we have

$$
\left\|D \exp \left(-t D^{*} D\right)\right\|=\left\|U \sqrt{H_{D}} \exp \left(-t H_{D}\right)\right\|=\left\|\sqrt{H_{D}} \exp \left(-t H_{D}\right)\right\|
$$

which is $<\infty$ for all $t>0$ by the spectral calculus.

\footnotetext{
${ }^{2}$ st-lim $\lim _{t \rightarrow \pm \infty}$ stands for the strong limit.
} 


\section{REFERENCES}

[1] Bei, F. \& Güneysu, B. \& Müller, J.: Scattering theory of the Hodge-Laplacian under a conformal perturbation. J. Spectr. Theory 7 (2017), no. 1, 235-267.

[2] Breuer, Jonathan, Last, Yoram, Stability of spectral types for Jacobi matrices under decaying random perturbations. J. Funct. Anal. 245 (2007), no. 1, 249-283.

[3] Colin de Verdière, Yves, Truc, Francoise Scattering theory for graphs isomorphic to a regular tree at infinity. J. Math. Phys. 54 (2013), no. 6, 063502, 24pp.

[4] P. Deift, R. Killip, On the absolutely continuous spectrum of one-dimensional Schrödinger operators with square summable potentials, Comm. Math. Phys. 203 (1999) 341-347.

[5] Demuth, Michael, On topics in spectral and stochastic analysis for Schrödinger operators. Recent developments in quantum mechanics (Poiana Brasov, 1989), 223-242, Math. Phys. Stud., 12, Kluwer Acad. Publ., Dordrecht, 1991.

[6] Demuth, Michael, Stollmann, Peter, Stolz, Günter, van Casteren, Jan, Trace norm estimates for products of integral operators and diffusion semigroups. Integral Equations Operator Theory 23 (1995), no. 2, 145-153.

[7] Güneysu, B. \& Thalmaier, A.: Scattering theory without injectivity radius assumptions and spectral stability for the Ricci flow. Preprint: arXiv:1709.01612.

[8] Hempel, R. \& Post, O. \& Weder, R.: On open scattering channels for manifolds with ends, J. Funct. Anal. 266 (2014), no. 9, 5526-5583.

[9] Hempel, R. \& Post, O.: On Open Scattering Channels for a Branched Covering of the Euclidean Plane. Preprint (2017). arXiv:1712.09147.

[10] Y. Higuchi, Y. Nomura, Spectral structure of the Laplacian on a covering graph, European J. Combin., Volume 30, Number 2 (2009), p. 570-585.

[11] M. Keller, Absolutely continuous spectrum for multi-type Galton Watson trees, Annales Henri Poincare, 13, (2012), 1745-1766.

[12] M. Keller, D. Lenz, S. Warzel, On the spectral theory of trees with finite cone type, Israel Journal of Mathematics, 194, (2013), 107-135.

[13] M. Keller, D. Lenz, S. Warzel, An invitation to trees of finite cone type: random and deterministic operators, Markov Process Related Fields, 21, (2015), no. 3, part 1, 557-574.

[14] R. Killip, Perturbations of one-dimensional Schrödinger operators preserving the absolutely continuous spectrum, Int. Math. Res. Not. 38 (2002) 2029-2061.

[15] A. Kiselev, Absolutely continuous spectrum of one-dimensional Schrödinger operators and Jacobi matrices with slowly decreasing potentials, Comm. Math. Phys. 179 (1996) 377-400.

[16] A. Klein, Absolutely continuous spectrum in the Anderson model on the Bethe lattice. Math. Res. Lett. 1 (1994), 399-407.

[17] Y. Last, B. Simon, Eigenfunctions, transfer matrices, and absolutely continuous spectrum of onedimensional Schrödinger operators, Invent. Math. 135 (1999) 329-367.

[18] Müller, W. \& Salomonsen, G.: Scattering theory for the Laplacian on manifolds with bounded curvature. J. Funct. Anal. 253 (2007), no. 1, 158-206.

[19] T. Nagnibeda, W. Woess, Random walks on trees with finite cone type, J. Theoret. Probab. 15 (2002), $383-422$.

[20] Reed, M. \& Simon, B.: Methods of modern mathematical physics. III. Scattering theory. Academic Press [Harcourt Brace Jovanovich, Publishers], New York-London, 1979.

[21] C. Remling, The absolutely continuous spectrum of one-dimensional Schrödinger operators with decaying potentials, Comm. Math. Phys. 193 (1998) 151-170.

[22] Stollmann, Peter Scattering by obstacles of finite capacity. J. Funct. Anal. 121 (1994), no. 2, 416-425.

[23] Weidmann, J.: Lineare Operatoren in Hilberträumen. Teil II. Anwendungen. Mathematische Leitfäden. B. G. Teubner, Stuttgart, 2003. 
Institut für Mathematik, Humboldt-Universität ZU Berlin, 12489 Berlin, Germany

E-mail address: gueneysu@math.hu-berlin.de

Institut für Mathematik, Universität Potsdam, 14476 Potsdam, Germany 